\title{
diffusion-îundarrentals
}

The Open-Access Journal for the Basic Principles of Diffusion Theory, Experiment and Application

\section{Entropic forces generated by grafted semi-flexible polymers}

\author{
Azam Gholami ${ }^{1}$, Jan Wilhelm ${ }^{2}$ and Erwin Frey ${ }^{2}$ \\ ${ }^{1}$ Hahn-Meitner-Institut, Abteilung Theorie, Glienicker Str. 100, D-14109 Berlin, \\ Germany, \\ 2 Arnold Sommerfeld Center for Theoretical Physics and CeNS, \\ Department of Physics, Ludwig-Maximilian-Universität München, \\ Theresienstrasse 37, D-80333 München, Germany
}

\section{INTRODUCTION}

The force exerted by a fluctuating semi-flexible polymer on a rigid obstacle and the probability to find a gap of given size between the wall and the polymer's tip are

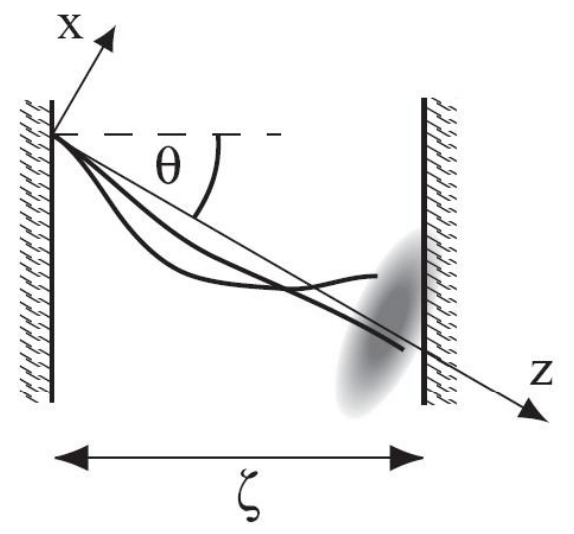
quantities of immediate interest for recent theories of force generation by action or microtubule polymerization [1,2]. Force generation of this type is thought to play a prominent role in cell motility. The fluctuating sections of filament are typically very short compared to their persistence length $\mathrm{p}=\kappa / k_{\mathrm{B}} \mathrm{T}$ where $\kappa$ is the polymers bending modulus. This allows the use of approximations which make the problem solvable up to quadrature for the general case.

FIG. 1: A smooth hard wall constraints the fluctuations of a grafted semi-flexible polymer with a contour length $L$ resulting in an average force between wall and graft. Two possible configurations of the polymer are indicated. The shaded area is a symbolic representation of the distribution of the loci of the polymer's end in the absence of the wall.

\section{RESULTS}

We compute the average force exerted by a fluctuating grafted semi-flexible polymer upon a rigid smooth wall as well as the corresponding free energy in $2 \mathrm{~d}$ and $3 \mathrm{~d}$. Both quantities are thought to be of interest for understanding the physics of actinpolymerization driven cell motility.

As Fig. (3) shows force in $2 \mathrm{~d}$ has a peak which means that somewhere the entropic force goes beyond mechanical one. We think this peak is just a geometrical effect due to restricting the polymer to fluctuate in $2 \mathrm{~d}$ which entropic forces are stronger than $3 \mathrm{~d}$. 

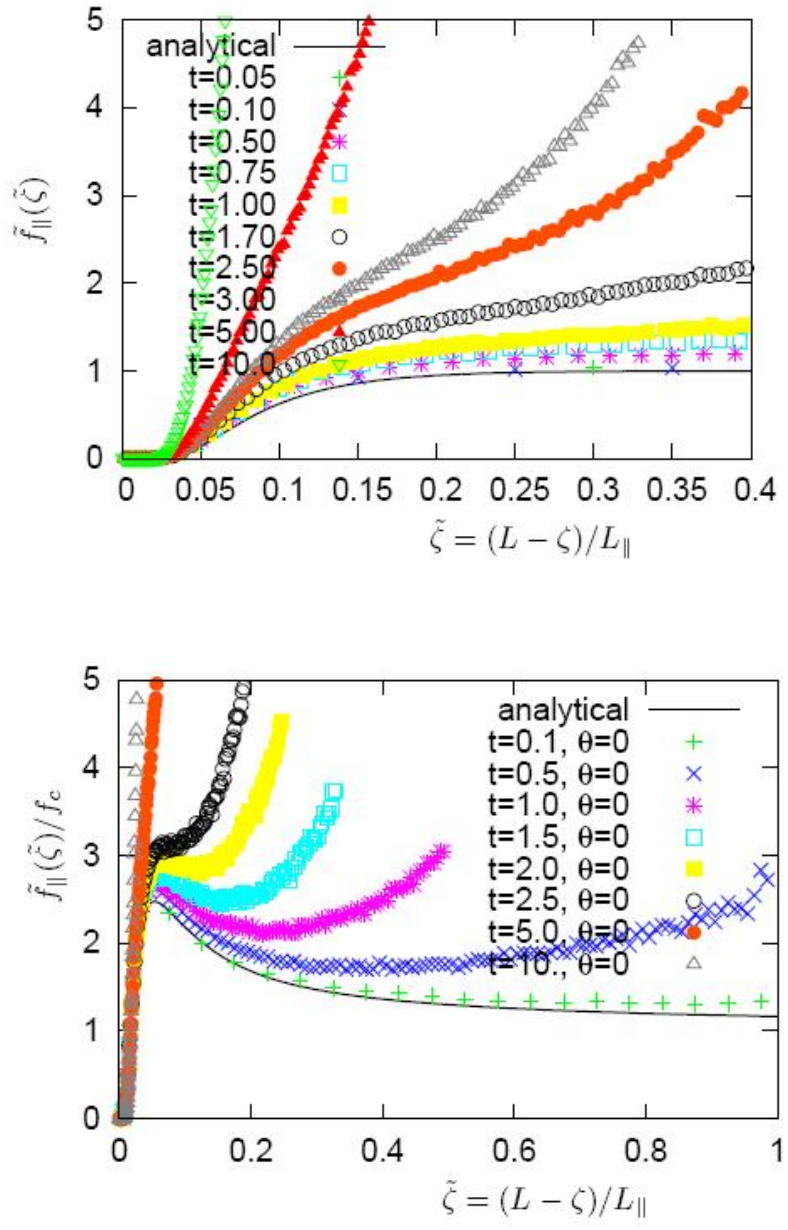

FIG. 2: Universal scaling function $f \|(\zeta)$ for the force exerted on a wall at a distance $\zeta$ from the grafted end in $3 d$ at $\theta=0$. The scaling variable $\zeta=(\mathrm{L}-\zeta) / \mathrm{L}_{\|}$measures the smallest allowed stored length $(\mathrm{L}-\zeta)$ in units of $\mathrm{L}_{\|}$ where $\mathrm{L}_{\|}=\mathrm{Lt}$ and $\mathrm{t}=\mathrm{L} / \mathrm{p}$.

FIG. 3: Average force between grafted polymer at $\theta=0$ and rigid wall for different values of $\mathrm{t}=\mathrm{L} / \mathrm{p}_{\mathrm{p}}$ in $2 \mathrm{~d}$ where $\mathrm{L}_{\|}=2 \mathrm{Lt}$.

Depending on the angle between the constraining wall and the direction of the graft, two asymptotic regimes with different dependence of the force on the position of the wall can be discerned. The angle determining the position of the crossover varies as the square root of the ratio of the polymer's length to its persistence length. For angles larger than the critical angle, previous expressions are qualitatively valid but for smaller angles different behavior is found.

\section{REFRENCES}

[1] A. Mogilner and G. Oster, Eur. Biophys. J. 25, 47 (1996).

[2] A. Mogilner and G. Oster, Biophys J. 71, 3030 (1996).

[3] Landau and Lifshitz, Theory of Elasticity, 7, of Course of Theoretical Physics (Pergamon Press, London, 1959).

[4] O. Kratky and G. Porod, Rev. Trav. Chim. 68, 1106 (1949)

[5] N. Saito, K. Takahashi, and Y. Yunoki, J. Phys. Soc. Jap. 22, 219 (1967)

[6] J. Wilhelm and E. Frey, Phys. Rev. Lett.77, 2581 (1996)

[7] K. Kroy and E. Frey, Phys. Rev. Lett. 77, 306 (1996)

Diffusion Fundamentals 3 (2005) 4.1 - 4.2 